\title{
Reseña de la búsqueda de hacer agujeros
}

\author{
José G. Anaya, David Maya, \\ Alejandro Fuentes-Montes De OCA* \\ Universidad Autónoma del Estado de México, Facultad de Ciencias, Toluca, México.
}

Resumen. Un espacio topológico conexo $Z$ es unicoherente si para cualesquiera $A$ y $B$ cerrados y conexos de $Z$, tales que $Z=A \cup B$, se tiene que $A \cap B$ es conexa. Sea $Z$ un espacio unicoherente: decimos que $z \in Z$ agujera a $Z$ si $Z-\{z\}$ no es unicoherente. Un problema de reciente estudio es: dado un espacio topológico unicoherente $\mathcal{H}(Z)$, obtenido de un espacio topológico $Z$, ¿cuáles elementos $A \in \mathcal{H}(Z)$ lo agujerean? Este trabajo consiste en dar una reseña de los resultados que hasta la fecha se conocen de este problema. Palabras clave: Continuo, hiperespacios, propiedad b), unicoherencia, cono, suspensión.

MSC2010: 54B15, 54B20, 54F55.

\section{Review of the search for holes}

Abstract. A connected topological space $Z$ is unicoherent provided that if $Z=A \cup B$, where $A$ and $B$ are closed connected subsets of $Z$, then $A \cap B$ is connected. Let $Z$ be a unicoherent space: we say that $z \in Z$ makes a hole in $Z$ if $Z-\{z\}$ is not unicoherent. A problem of recent study is: given a topological space unicoherent $\mathcal{H}(Z)$, obtained from a topological space $Z$, which elements $A \in \mathcal{H}(Z)$ makes a hole? This work consists in giving a review of the results known to date of this problem.

Keywords: Continuum, hyperspaces, property b), unicoherence, cone, suspension.

\section{Introducción}

La unicoherencia es una propiedad topológica que surge de trabajos de distintos autores, entre los cuales podemos mencionar a L. E. J. Brouwer (1910), F. Hausdorff (1914), Z. Janiszewsky (1913), S. Mazurkiewicz (1922) y R. L. Moore (1924). El primero en definirla y llamarla tal y como la conocemos fue K. Kuratowski (ver [21] y [22]). En [11], K.

\footnotetext{
*E-mail: afuentesm@uaemex.mx

Recibido: 12 de octubre de 2017, Aceptado: 8 de agosto de 2018.

Para citar este artículo: J.G. Anaya, D. Maya, A. Fuentes-Montes de Oca, Reseña de la búsqueda de hacer agujeros, Rev. Integr. temas mat. 36 (2018), No. 2, 101-116. doi: 10.18273/revint.v36n2-2018003.
} 
Borsuk introduce el uso de funciones continuas de un espacio al círculo unitario, y con esto encuentra una nueva forma de estudiar a la unicoherencia de un espacio topológico. Un excelente trabajo que podemos recomendar acerca de la unicoherencia es [17].

De manera intuitiva podemos decir que un espacio es unicoherente si no tiene "agujeros". Podemos pensar que un espacio topológico conexo $Z$ tiene un "agujero", si podemos "cubrir" con dos cerrados y conexos a $Z$ de tal forma que su intersección sea no conexa. Con esta idea intuitiva podemos ver que el círculo unitario en el plano y el toro son ejemplos de espacios que tienen un "agujero". Siguiendo de manera intuitiva, podemos preguntarnos: ¿qué le pasa a un espacio topológico unicoherente si le quitamos un elemento? Si pierde la propiedad de la unicoherencia entonces vemos que "agujerea". Esta es básicamente la idea que abrió paso a una nueva rama de la Teoría de Continuos y sus Hiperespacios.

Durante todo el trabajo nos referiremos al autor o los autores de los resultados que se irán mencionando, evitando así llenar la lectura con los nombres de ellos. Dividimos al trabajo en cinco partes. La primera es esta en la que estamos. En la segunda parte damos las definiciones y resultados que necesitamos en el desarrollo del trabajo. En la tercera parte mostramos las herramientas que se utilizan en las demostraciones, y mostramos cómo se relacionan los conceptos de propiedad b) y de unicoherencia. En la cuarta parte se enuncian los resultados que se conocen de la búsqueda de los elementos que agujerean un espacio, y en cada resultado hacemos una descripción sistemática de la prueba que presentan los autores. Finalmente, la quinta parte consiste de las conclusiones de este trabajo.

\section{Conceptos generales}

Vamos a representar el conjunto de los enteros positivos como $\mathbb{N}$, el conjunto de los números reales como $\mathbb{R}$, al intervalo $[0,1]$ como $I$, a la $n$-celda como $I^{n}$ (para $\left.n \in \mathbb{N}\right)$, al cubo de Hilbert $I \times I \times \ldots$ como $I^{\infty}$ y a la $n$-esfera como $S^{n}($ para $n \in \mathbb{N})$. La cardinalidad de un conjunto $A$ la denotaremos por $|A|$.

Sea $Z$ un espacio topológico y $A \subset Z$; denotaremos por $c l(A)$ la cerradura de $A, b d(A)$ la frontera de $A$ y $\operatorname{int}(A)$ el interior de $A$, todos estos en $Z$. A los elementos de $\operatorname{int}(A)$ se los llama puntos interiores. Dados dos espacios topológicos $Y$ y $Z$ formamos el espacio producto $Y \times Z$, con la topología producto. Otro espacio que formaremos a partir espacios topológicos $Y$ y $Z$ es el espacio cociente, y lo escribiremos como $Y / Z$, dotado con la topología cociente. Cuando dos espacios topológicos $Y$ y $Z$ sean homeomorfos, lo denotaremos como $Y \approx Z$.

Una variedad de dimensión $n$ o $n$-variedad es un espacio de Hausdorff $M$ en el cual cada uno de sus puntos tiene una vecindad homeomorfa a $\mathbb{R}^{n}$. Una $n$-variedad compacta se llama cerrada. Una $n$-variedad con frontera es un espacio de Hausdorff $M$ en el cual cada uno de sus puntos tiene una vecindad homeomorfa a $\mathbb{R}^{n}$ o a $\mathbb{R}_{+}^{n}=\left\{\left(x_{1}, \ldots, x_{n}\right) \in\right.$ $\left.\mathbb{R}^{n}: x_{n} \geq 0\right\}$.

Una propiedad topológica $P$ es hereditaria en $Z$, si cada subespacio de $Z$ tiene la propiedad en cuestión. Al referirnos como " $Z$ hereditariamente $P$ ", será porque $P$ es hereditaria en $Z$.

Un espacio topológico $X$ es un continuo si es un espacio métrico compacto conexo no degenerado, con métrica $d$. A lo largo del trabajo, $X$ siempre denotará un continuo.

[Revista Integración, temas de matemáticas 
Diremos que un subespacio $Y \subset X$ es un subcontinuo de $X$, si es por sí mismo un continuo.

Un arco es un espacio topológico homeomorfo a $I$. Un arco $p q$ en un espacio topológico $Z$, con puntos finales $p$ y $q$, es llamado arco libre si $p q-\{p, q\}$ es un subconjunto abierto en $Z$. Sabemos que una curva cerrada simple es un espacio topológico homeomerfo a $S^{1}$. Una curva cerrada simple $S$ es llamada curva cerrada simple libre en $Z$ si $S \neq Z$ y existe $p \in S$ tal que $S-\{p\}$ es un subconjunto abierto de $Z$

Un espacio topológico $Z$ es localmente conexo si para cualquier $x \in Z$ y una vecindad abierta $U$ de $x$ existe un conjunto abierto conexo $C$ tal que $x \in C \subset U$. Un espacio de Peano es un espacio localmente conexo.

Sea $Y$ un subespacio de un espacio topológico $Z$. Una retracción de $Z$ en $Y$ es una función continua $r: Z \rightarrow Y$ tal que, para toda $y \in Y, r(y)=y$. Decimos que $Y$ es un retracto por deformación de $Z$ si existen una retracción $r: Z \rightarrow Y$ y una función continua $H: Z \times I \rightarrow Z$ tales que para cada $z \in Z$ tenemos: $H(z, 0)=z$ y $H(z, 1)=r(z)$. Decimos que un espacio topológico $Z$ es contráctil si existe $z \in Z$, tal que $\{z\}$ es un retracto por deformación de $Z$.

Un elemento $z$ en un espacio topológico conexo $Z$ es un punto de corte de $Z$ si $Z-\{z\}$ no es conexo. El conjunto de puntos de corte de $Z$ se denota por $C u t(Z)$. Un punto final $z \in Z$ es un elemento tal que no es punto de corte de un arco que lo contenga. Consideremos $E(Z)$ el conjunto de puntos finales. Un elemento $z$ de $Z$ es un punto de ramificación, siempre que $z$ sea un punto final de tres o más arcos en $Z$. El conjunto de todos los puntos de ramificación se denota por $R(Z)$.

Un continuo $X$ es un abanico si es arcoconexo, hereditariamente unicoherente y con exactamente un punto de ramificación. A ese punto de ramificación se lo llama vértice del abanico. Un continuo de Peano $X$ se dice que es una dendrita si no contiene curvas cerradas simples. Una gráfica finita es un continuo que se compone de un número finito de arcos, tales que cualesquiera dos de ellos son ajenos, o se intersecan en un solo punto o en sus puntos finales.

Una compactación del rayo $R=[0, \infty)$ con residuo un arco $J$ es llamada un Elsa continuo, y se denota $X=J \cup R$. Esta clase de continuos fue definida por S. B. Nadler Jr., en [28]. Se sabe que existe una cantidad no numerable de Elsa continuos (ver [26, p. 184]), entre los cuales se encuentra el conocido $\sin (1 / x)$-continuo. A partir de esta familia podemos construir otras dos familias no numerables de continuos. La primer familia es la de los Elsa círculos (ver [5, Definition 4.19]): se considera un Elsa continuo $J \cup R$ y se identifica un extremo de $J$ con el extremo $R$. Un ejemplo de este es el círculo de Varsovia. La segunda es la de los doble Elsa círculos (ver [5, Definition 4.20]): se considera la compactación de dos rayos con el mismo residuo $J$ y los extremos de los rayos se identifican.

Los conceptos que no mencionamos en esta sección y que lleguemos a usar se definirán en el lugar adecuado.

\section{Unicoherencia y Propiedad b)}

Iniciemos con el siguiente concepto, que es una herramienta muy útil en la búsqueda de agujeros. Sea $Z$ un espacio topológico. Decimos que $Z$ tiene la propiedad b) si para cada

Vol. 36, $\left.\mathrm{N}^{\circ} 2,2018\right]$ 
función continua $f: Z \rightarrow S^{1}$, existe una función continua $h: Z \rightarrow \mathbb{R}$ tal que $f=\exp \circ h$, donde exp es la función de $\mathbb{R}$ sobre $S^{1}$ definida por $\exp (t)=(\cos (2 \pi t), \sin (2 \pi t))$. A la función $h$ se la llama levantamiento de $f$.

Esta sección tiene dos propósitos. El primero es mostrarle al lector algunos de los resultados que sirven como herramientas para la búsqueda de agujeros. El segundo es mostrar la relación que hay entre la propiedad b) y la unicoherencia.

Proposición 3.1 ([1, Proposition 8, p. 2001]). Sea $Z$ un espacio topológico tal que $Z=$ $A \cup B$, donde $A$ y $B$ son cerrados y conexos. Si $A$ y $B$ tienen la propiedad b) y $A \cap B$ es conexo, entonces $Z$ tiene la propiedad b).

El siguiente resultado fue probado por S. Mardešić (1958).

Teorema 3.2 ([25, Lemma 5, p. 39]). Sea $Z$ un espacio topológico y $f: Z \rightarrow S^{1}$ una función continua. Entonces $f$ tiene un levantamiento si, y sólo si, $f$ es homotópica a una función constante.

Proposición 3.3. Sea $Z$ un espacio topológico y sea $Y$ un retracto por deformación de $Z$. Entonces $Z$ tiene la propiedad b) si, y sólo si, $Y$ tiene la propiedad b).

Demostración. Dado que $Y$ es un retracto por deformación de $Z$, existen una retracción $r: Z \rightarrow Y$ y una función continua $H: Z \times I \rightarrow Z$ tales que para cada $z \in Z$ tenemos $H(z, 0)=z$ y $H(z, 1)=r(z)$.

Probemos la necesidad: sea $f: Y \rightarrow S^{1}$ una función continua. Como $Z$ tiene la propiedad b), existe un levantamiento $h: Z \rightarrow \mathbb{R}$ de $f \circ r$. Consideremos $\left.h\right|_{Y}: Y \rightarrow \mathbb{R}$. Dado $y \in Y$, tenemos que exp $\left.\circ\right|_{Y}(y)=f \circ r(y)=f(y)$. Esto prueba que $f$ tiene un levantamiento, y por tanto $Y$ tiene la propiedad b).

Para la suficiencia, consideremos una función continua $f: Z \rightarrow S^{1}$. Probaremos que $f$ es homotópica a una constante. Dado que $Y$ tiene la propiedad b), por el Teorema 3.2, $\left.f\right|_{Y}$ es homotópica a una constante. De manera que existen $s_{0} \in S^{1}$ y una función $F: Y \times I$ $\rightarrow S^{1}$ tales que, para cada $y \in Y, F(y, 0)=\left.f\right|_{Y}(y)$ y $F(y, 1)=s_{0}$. Definimos $F_{1}: Z \times I$ $\rightarrow S^{1}$ por

$$
F_{1}(z, t)=\left\{\begin{array}{cc}
f(H(z, 2 t)), & \text { si } t \in[0,1 / 2], \\
F(r(z), 2 t-1), & \text { si } t \in[1 / 2,1]
\end{array} .\right.
$$

Como $f(H(z, 1))=f(r(z))$ y $F(r(z), 0)=f(r(z))$, para toda $z \in Z$, tenemos que $F_{1}$ es continua. Además, para cada $z \in Z$ tenemos que $F_{1}(z, 0)=f(H(z, 0))=f(z)$ y $F_{1}(z, 1)=F(r(z), 1)=s_{0}$. De tal manera que $f$ es homotópica a una constante y por el Teorema $3.2 f$ tiene un levantamiento, así concluimos que $Z$ tiene la propiedad b).

Proposición 3.4. Cada espacio topológico contráctil tiene la propiedad b).

Demostración. Si $Z$ es contráctil, entonces existe $z \in Z$ tal que $\{z\}$ es un retracto por deformación de $Z$. Dado que $\{z\}$ tiene la propiedad b), por la Proposición 3.3, $Z$ tiene la propiedad b).

Ahora hablaremos de la unicoherencia y la relación que tiene con la propiedad b).

[Revista Integración, temas de matemáticas 
Teorema 3.5 ([14, Théorème 2 and 3, p. 69 and 70] o [30, Theorem 7.3, p. 227]). Sea $Z$ un espacio topológico normal. Si $Z$ tiene la propiedad b), entonces es unicoherente.

Ejemplo 3.6. Tenemos que el intervalo $I$ tiene la propiedad b); resulta ser un agradable ejercicio probar que esto es verdad. La circunferencia $S^{1}$ no la tiene; para ver esto se puede probar que la identidad $i_{S^{1}}: S^{1} \rightarrow S^{1}$ no tiene un levantamiento. Una familia de continuos que tiene la propiedad b) es la de los Elsa continuos (ver [27, 12.66, p. 269]) y por el Teorema 3.5 es unicoherente. Por otro lado, es posible probar directamente que el intervalo $I$ es unicoherente, mientras que la circunferencia $S^{1}$ no es unicoherente.

Observación 3.7. La implicación en la otra dirección del Teorema 3.5 no siempre es cierta; un ejemplo de esto es $X=S^{1} \cup\{[1+1 / t] \exp (i t): t \geq 1\}$, representado en el plano complejo. Este continuo es una compactación del rayo $R=[0, \infty)$ con residuo $S^{1}$. Al estudiar este continuo, podemos ver que sólo hay cuatro tipos de subcontinuos: puntos, arcos, $S^{1}$ y homeomorfos a $X$. Con esto es fácil probar que $X$ es unicoherente. Por otro lado, se puede probar que una función $f: X \rightarrow S^{1}$, tal que $\left.f\right|_{S^{1}}=i_{S^{1}}$, no tiene levantamiento.

Existe una equivalencia para estas dos propiedades.

Teorema 3.8 ([13, Theorem 3, p. 70]). Sea $Z$ es un espacio topológico $T_{1}$, normal y localmente conexo. $Z$ es unicoherente si, y sólo si, $Z$ tiene la propiedad b).

Sean $Y$ y $Z$ dos continuos. Sea $f: Y \rightarrow Z$ una función continua; decimos que $f$ es monótona si para cada $y \in Y$ se tiene que $f^{-1}(y)$ es un continuo.

Teorema 3.9 ([30, Theorem 2.21, p. 138]). La unicoherencia es invariante bajo funciones monótonas entre continuos.

Teorema 3.10 ([30, Corollary 7.41, p. 228]). La propiedad b) es invariante bajo funciones monótonas entre continuos localmente conexos.

Teorema 3.11 ([30, Theorem 7.5, p. 228]). Si dos continuos $Z$ y $Y$ tienen la propiedad b), entonces $Z \times Y$ también la tiene.

Teorema 3.12. Sea $Y$ un espacio conexo. Si $X \times Y$ es unicoherente, entonces $X$ es unicoherente.

Demostración. Supongamos que $X \times Y$ es unicoherente. Consideremos la proyección $\pi_{X}: X \times Y \rightarrow X$ dada por $\pi_{X}(x, y)=x$ para cualquier $(x, y) \in X \times Y$. Nótese que $\pi_{X}$ es monótona. Por [17, Theorem 3.6, p. 40] tenemos que $\pi_{X}(X \times Y)=X$ es unicoherente. $\square$

El recíproco del teorema anterior no se sabe si es cierto; de hecho pertenece a un problema abierto más general del cual se sabe en algunos casos. K. Kuratowsky (1930) propuso el problema: ¿es unicoherente el producto de dos continuos unicoherentes de Peano? Bursuk en [11] contestó afirmativamente este problema. S. Eilenberg [14] dio una prueba para un caso más general: el producto de dos espacios métricos unicoherentes localmente conexos es unicoherente. Fue T. Ganea en [15, Theorem 1.3, p. 35] que dio una generalización de esto: el producto de una familia arbitraria de espacios unicoherentes localmente conexos es unicoherente. Se sabe que el continuo $X$ de la observación 3.7 cumple que $X \times X$ no es unicoherente (ver [17, Example 5.5, p. 48 and 49]).

Vol. 36, $\left.\mathrm{N}^{\circ} 2,2018\right]$ 


\section{Búsqueda de agujeros}

Consideremos los siguientes espacios topológicos $\mathcal{H}(X)$ :

1. $2^{X}=\{A \subset X: A$ es cerrado y no vacío $\}$, el hiperespacio de los cerrados de $X$.

2. $C_{n}(X)=\left\{A \in 2^{X}: A\right.$ tiene a lo más $n$ componentes $\}$, el hiperespacio de los cerrados con a lo más $n$ componentes de $X$.

3. $F_{n}(X)=\left\{A \in 2^{X}: A\right.$ tiene a lo más $n$ elementos $\}$, el $n$-ésimo producto simétrico de $X$.

4. Cono $(X)=(X \times I) /(X \times\{1\})$, el cono topológico de $X$.

5. Sus $(X)=\operatorname{Cono}(X) /(X \times\{0\})$, la suspensión topológica de $X$.

Si $n=1$, entonces a $C_{1}(X)$ lo escribiremos como $C(X)$ y lo llamaremos el hiperespacio de subcontinuos, a $F_{1}(X)$ lo llamaremos el hiperespacio de los unipuntuales y a $F_{2}(X)$ lo llamaremos el segundo producto simétrico de $X$. A los primeros tres los consideramos con la métrica de Hausdorff (ver [20]). En los últimos dos se consideran la topología producto y la topología cociente.

Presentamos ahora la definición de nuestro interés: sea $Z$ un espacio topológico unicoherente y sea $z \in Z$. Decimos que $z$ agujera (o hace agujero) en $Z$ si $Z-\{z\}$ no es unicoherente.

Para ilustrar la importancia de la busqueda de elementos que agujeran, mencionamos el siguiente caso que sirvió para distinguir diferencias entre espacios topológicos. En [19, lemmas 2.1 and 2.2 , p. 348 and 349] A. Illanes probó que $C_{2}([0,1])-\{A\}$ es unicoherente para cada $A \in C_{2}([0,1])$, mientras que $C_{2}\left(S^{1}\right)-\left\{S^{1}\right\}$ no es unicoherente. Como consecuencia, A. Illanes obtuvo que $C_{2}([0,1])$ y $C_{2}\left(S^{1}\right)$ no son homeomorfos; esto contrasta con el hecho de que $C([0,1])$ y $C\left(S^{1}\right)$ son homeomorfos: ambos son homeomorfos a $I^{2}$ (ver [20, Example 5.1 y Example 5.2, p. 33-36]). Este trabajo es el primero en donde se estudia la unicoherencia de un espacio topológico sin un elemento, aunque no es ahí en donde se le da el nombre. El primer trabajo que aborda el problema de manera directa y que define el concepto es [1].

Por definición vemos que para buscar elementos que agujeren un espacio debemos tener necesariamente que este espacio sea unicoherente. En [29, Corollary 1.176, p. 178] S. B. Nadler Jr. probó que los hiperespacios $2^{X}$ y $C(X)$ son unicoherentes para cualquier $X$. En [24] S. Macías probó que el hiperespacio $C_{n}(X)$ tiene la propiedad b) y que es unicoherente, para cualquier $X$ y para todo $n \geq 1$. El mismo autor en [23] probó que el hiperespacio $F_{n}(X)$ es unicoherente, para cualquier $X$ y para todo $n \geq 3$. Para el cono y la suspensión tenemos lo siguiente. Al considerar a $Z$ como espacio métrico, tenemos que $\operatorname{Cono}(Z)$ y $\operatorname{Sus}(Z)$ son espacios normales (ver [5, Proposition 3.2]). Por otro lado, se prueba con cierta facilidad que si $v$ es el vértice del cono, entonces $\{v\}$ es un retracto por deformación de $\operatorname{Cono}(Z)$, así que $\operatorname{Cono}(Z)$ es contráctil. Por la Proposición 3.4 tenemos que $\operatorname{Cono}(Z)$ tiene la propiedad b), y en consecuencia es unicoherente por el Teorema 3.5. Ahora, por [17, Theorem 3.6, p. 40] tenemos que $S u s(X)$ es unicoherente. Como habrá notado el lector, el caso de $F_{2}(X)$ es uno aparte. Se sabe que $F_{2}\left(S^{1}\right)$ es homeomorfo a la 
Banda de Moebius (ver [20, Exercise 1.26, p. 9]), la cual no es unicoherente, como es fácil de ver. También se conoce un continuo que es unicoherente, pero cuyo segundo producto simétrico no lo es (ver [12]). Esto lleva a estudiar la generalización de la unicoherencia en este hiperespacio (ver las conclusiones al final).

Antes de iniciar con la descripción de los resultados vamos a ver un poco de las herramientas que se usan en los hiperespacios.

Una función continua $\mu: 2^{X} \rightarrow[0, \infty)$ es llamada una función de Whitney si $\mu(\{p\})=0$ para $p \in X$ y $\mu(A)<\mu(B)$ si $A \subset B$. Si agregamos la condición $\mu(X)=1$ podemos definir a la función sobre $I$. Además podemos hacer la restricción a $C(X)$. La existencia de funciones de Whitney se garantiza en [20, Theorem 13.4, p. 107]. Un nivel de Whitney es un subconjunto de $C(X)$ de la forma $\mu^{-1}(t)$ para $t \in I$. Un bloque de Whitney es un subconjunto de $C(X)$ de la forma $\mu^{-1}([a, b])$ para $[a, b] \subset I$. Se sabe que los niveles y bloques de Whitney son sobcontinuos de $C(X)$ (ver [20, Theorem 19.9, p. 160]).

\subsection{Para $C(X)$}

En [1] el autor prueba los siguientes resultados.

Teorema 4.1 ([1, Theorem 1, p. 2001]). Si pq es un arco libre en $X$ tal que $p$ y q no son puntos interiores de $p q$, entonces pq agujera a $C(X)$.

Demostración. Sea $Y=X-i n t(p q)$. Consideremos los siguientes subconjuntos de $C(X)$ : $\mathcal{D}_{1}=\{A \in C(X)-\{p q\}: A \subset p q\}$ y $\mathcal{D}_{2}=\{B \in C(X)-\{p q\}: B \cap Y \neq \emptyset\}$. Tenemos que $\mathcal{D}_{1}$ y $\mathcal{D}_{2}$ son subconjuntos cerrados y conexos de $C(X)-\{p q\}$, y además $\mathcal{D}_{1} \cup \mathcal{D}_{2}=$ $C(X)-\{p q\}$. Veamos qué sucede con la intersección. Sean $\mathcal{E}_{1}=\{p r \subset p q: r \in p q-\{q\}\}$ y $\mathcal{E}_{2}=\{t q \subset p q: t \in p q-\{p\}\}$. Es fácil ver que $\mathcal{E}_{1} \cap \mathcal{E}_{2}=\emptyset$. Como $\mathcal{D}_{1} \cap \mathcal{D}_{2}=\mathcal{E}_{1} \cup \mathcal{E}_{2}$, podemos concluir que $C(X)-\{p q\}$ no es unicoherente, y por lo tanto $p q$ agujera a $C(X)$.

Teorema 4.2 ([1, Theorem 2, p. 2001]). Si $X$ es un continuo localmente conexo y $X$ no es una curva cerrada simple, entonces $X$ no agujera a $C(X)$.

Demostración. Consideremos una función de Whitney $\mu: C(X) \rightarrow I$. Por [18, Theorem D, p. 253] tenemos que existe $s<1$ tal que $\mu^{-1}(s)$ es unicoherente. Por [18, Theorem A, p. 252] tenemos que $\mu^{-1}(t)$ es unicoherente para cada $t \in[s, 1]$. Ahora, por [29, Theorem 14.9 , p. 408] tenemos que $\mu^{-1}(t)$ es localmente conexo para cada $t \in I$. Por Teorema 3.8 tenemos que $\mu^{-1}(t)$ tiene la propiedad $\mathrm{b}$ ), para cada $t \in[s, 1]$. Se puede probar que $\mu^{-1}(t)$ es un retracto por deformación de $\mu^{-1}([0, t])$ (el lector interesado puede usar la función continua definida en $[29,0.65 .1$, p. 37]). Así, por la Proposición 3.3 tenemos que $\mu^{-1}([0, t])$ tiene la propiedad b) para $t \in[s, 1]$.

Sea $f:(C(X)-\{X\}) \rightarrow S^{1}$ una función continua. Fijemos a un punto $x_{0} \in X$ y a un número $t_{0} \in \exp ^{-1}\left(f\left(\left\{x_{0}\right\}\right)\right)$. Sea $\left\{t_{n}\right\}$ una sucesión real estrictamente creciente tal que $t_{1}=s$ y $\left\{t_{n}\right\} \rightarrow 1$. Para cada $n \in \mathbb{N}$, sea $f_{n}=\left.f\right|_{\mu^{-1}\left(\left[0, t_{n}\right]\right)}$. Para cada $n \in \mathbb{N}$, considere $h_{n}: \mu^{-1}\left(\left[0, t_{n}\right]\right) \rightarrow \mathbb{R}$ el único levantamiento de $f_{n}$ tal que $h_{n}\left(\left\{x_{0}\right\}\right)=t_{0}$. Definamos ahora $h: \bigcup_{n=1}^{\infty} \mu^{-1}\left(\left[0, t_{n}\right]\right) \rightarrow \mathbb{R}$ dada por $h(A)=h_{n}(A)$, si $A \in \mu^{-1}\left(\left[0, t_{n}\right]\right)$. No es 
díficil ver que $h$ está bien definida y que es continua. También es sencillo ver que si $A \in$ $\bigcup_{n=1}^{\infty} \mu^{-1}\left(\left[0, t_{n}\right]\right)$, entonces $h(A)=\exp (f(A))$. Dado que $C(X)-\{X\}=\bigcup_{n=1}^{\infty} \mu^{-1}\left(\left[0, t_{n}\right]\right)$, podemos concluir que $h$ es un levantamiento de $f$. Por lo tanto $X$ no agujera a $C(X)$.

Si $X \approx S^{1}$, entonces $X \in \operatorname{int}(C(X))$ considerada como una 2-variedad (ver [20, Example 5.2 , p. 35]). En este caso tenemos que $X$ agujera a $C(X)$.

Sea $\mathcal{H} \in\left\{2^{X}, C(X)\right\}$ y sea $\mathcal{K}_{A}=\{B \in \mathcal{H}: A \subset B\}$, para $A \in \mathcal{H}$.

Lema 4.3 ([1, Lemma 13, p. 2004]). Sea $\mathcal{K}$ un subconjunto no vacío de $\mathcal{H}$. Si $\mathcal{K}_{A} \subset \mathcal{K}$, para cada $A \in \mathcal{K}$, entonces $\mathcal{K}$ tiene la propiedad $b$ ).

La prueba del siguiente resultado se sigue de manera natural del lema anterior, considerando a $\mathcal{K}=\mathcal{H}-\{x\}$.

Teorema 4.4 ([1, Theorem 3, p. 2001]). Si $x \in X$, entonces $\{x\}$ no agujera a $2^{X} y\{x\}$ no agujera a $C(X)$.

Para la demostración del siguiente teorema el autor usa la siguiente afirmación: Sea $p$ un elemento de $X$ y $Y_{1}, Y_{2} \in C(X)$ tales que $Y_{1}, Y_{2}$ son no degenerados, $X=Y_{1} \cup Y_{2}$ y $Y_{1} \cap Y_{2}=\{p\}$. Entonces existe una función de Whitney $\mu: C(X) \rightarrow I$ tal que $\mu\left(Y_{1}\right)<$ $\mu\left(Y_{2}\right)$ y $\mu^{-1}\left(\mu\left(Y_{1}\right)\right)-\left\{Y_{1}\right\}$ es conexo. (Ver [1, Lemma 14, p. 2005]).

Teorema 4.5 ([1, Theorem 4, p. 2001]). Si pq es un arco libre en $X$ tal que $p$ no es un punto interior de $p q$ y q es un punto interior de $p q$, entonces $p q$ no agujera a $C(X)$.

Demostración. Sea $f:(C(X)-\{p q\}) \rightarrow S^{1}$ una función continua. Probando que $f$ tiene un levantamiento podemos concluir que $C(X)-\{p q\}$ tiene la propiedad b). Sea $Y=X-i n t(p q)$. Notemos que $X=Y \cup p q$ y $Y \cap p q=\{p\}$. Así que $Y$ es conexo (ver [1, Proposition 7, p. 2001]). Por lo tanto $p q$ y $Y$ cumplen las condiciones de la afirmación previa a este teorema, y tenemos que existe una función de Whitney $\mu: C(X) \rightarrow I$ tal que $\mu^{-1}(\mu(p q))-\{p q\}$ es conexa. Sea $t_{0}=\mu(p q)$. Consideremos las siguientes funciones $f_{1}=\left.f\right|_{\mu^{-1}\left(\left[t_{0}, 1\right]\right)-\{p q\}}$ y $f_{2}=\left.f\right|_{\mu^{-1}\left(\left[0, t_{0}\right]\right)-\{p q\}}$. Ahora se puede probar que existen funciones continuas $g_{1}:\left(\mu^{-1}\left(\left[t_{0}, 1\right]\right)-\{p q\}\right) \rightarrow \mathbb{R}$ y $g_{2}:\left(\mu^{-1}\left(\left[0, t_{0}\right]\right)-\{p q\}\right) \rightarrow \mathbb{R}$, tales que $f_{1}=\exp \circ g_{1}, f_{2}=\exp \circ g_{2}$ y $\left.g_{1}\right|_{\mu^{-1}\left(t_{0}\right)-\{p q\}}=\left.g_{2}\right|_{\mu^{-1}\left(t_{0}\right)-\{p q\}}$. Ya para finalizar se define la función $g:(C(X)-\{p q\}) \rightarrow \mathbb{R}$ dada por

$g(A)= \begin{cases}g_{1}(A), & \text { si } A \in \mu^{-1}\left(\left[0, t_{0}\right]\right), \\ g_{2}(A), & \text { si } A \in \mu^{-1}\left(\left[t_{0}, 1\right]\right) .\end{cases}$

Entonces $g$ es un levantamiento de $f$, y con esto se concluye la prueba.

Teorema 4.6 ([1, Theorem 5, p. 2001]). Si S es una curva cerrada libre en $X$, entonces $S$ agujera a $C(X)$.

Demostración. Sea $S$ una curva cerrada libre, e identifiquémosla con el círculo unitario de $\mathbb{R}^{2}$. Como suponemos que $S$ es libre, existe $p \in S$ tal que $p \notin \operatorname{int}(S)$ y $S-\{p\}$ es un 
subconjunto abierto de $X$. Denotemos como $p_{0}$ el punto diametralmente opuesto a $p, \mathrm{y}$ tomemos un punto fijo $p_{1} \in S-\left\{p, p_{0}\right\}$. Sea $p_{0} p_{1}$ el arco en $S$ que cumple que $p \notin p_{0} p_{1}$.

Definamos la función $m: C(S)-\{S\} \rightarrow S$ tal que $m(B)$ es el punto de $B$ que lo divide en dos subarcos de igual longitud, siempre que $B$ sea un arco. Si $B$ es un singular, entonces $m(B)=B$.

Sean

$\mathcal{S}=\{A \in C(X)-\{S\}: A \cap S \neq \emptyset\} \mathrm{y}$

$\mathcal{F}=\left\{A \in \mathcal{S}: m(A \cap S) \in\left(S-\operatorname{int}\left(p_{0} p_{1}\right)\right)\right.$ y $\left.S \nsubseteq A\right\} \cup\{A \in \mathcal{S}: S \subset A\}$.

Dado que $\mathcal{S}$ es un subconjunto cerrado de $C(X)-\{S\}$, por [1, Lemma 15, p. 2006] tenemos que $\mathcal{F}$ es un subconjunto cerrado conexo de $C(X)-\{S\}$. Por otro lado, por [1, Proposition 7, p. 2001] tenemos que $Y=X-\operatorname{int}(S)$ es conexo y por lo tanto un subcontinuo de $X$. Más aún, $C(Y)$ es cerrado en $C(X)-\{S\}$. Entonces $\mathcal{G}_{1}=\mathcal{F} \cup C(Y)$ es cerrado en $C(X)-\{S\}$. También se puede ver que es conexo.

Definamos ahora

$\mathcal{G}_{2}=\left\{A \in \mathcal{S}: m(A \cap S) \in p_{0} p_{1}\right.$ y $\left.S \not A\right\} \cup\{A \in \mathcal{S}: S \subset A\}$.

Tenemos que $\mathcal{G}_{2}$ es un cerrado conexo de $C(X)-\{S\}$. De aquí es sencillo ver que $C(X)-$ $\{S\}=\mathcal{G}_{1} \cup \mathcal{G}_{2}$, y que $\mathcal{G}_{1} \cap \mathcal{G}_{2}$ no es conexo. Por lo tanto, $C(X)-\{S\}$ no es unicoherente. $\square$

Con respecto a los Teoremas 4.1 y 4.5, el autor hace notar que no se considera el caso cuando $p$ y $q$ son puntos interiores del arco libre $p q$. En este caso tenemos que $p q$ es abierto y cerrado en $X$, así $X$ es un arco y $C(X)$ es una 2-celda (ver [20, Example 5.1, p. 33]). Como $X \in b d(C(X))$ considerada como 2-variedad, tenemos que $p q$ no agujera a $C(X)$.

En [2] el autor da la clasificación de los elementos que agujeran a $C(X)$ cuando $X$ es un continuo localmente conexo. El teorema de clasificación es el siguiente.

Teorema 4.7 ([2, Theorem 7.1, p. 13]). Supongamos que $X$ es localmente conexo y sea $A$ un subcontinuo de $X$. Tenemos que $A$ agujera a $C(X)$ si, y sólo si, $A$ es de una de las siguientes formas:

1. $A=X$ y $X$ es una curva cerrada simple;

2. A es una curva cerrada simple libre, o

3. $A=p q$ es un arco libre tal que $p, q \notin \operatorname{int}(A)$.

Demostración. Probemos primero la necesidad. Sea $A$ un subcontinuo de $X$ y supongamos que $A$ agujera a $C(X)$. Si $A=X$, por el Teorema 4.2 tenemos que $X$ es una curva cerrada. Supongamos que $A \neq X$. Por $[2$, Proposition 6.2, p. 12] $A$ es localmente conexo. Ahora, por $[2$, Proposition 3.5, p. 8] $A$ es una curva cerrada simple o es un arco libre. Si es el caso de que sea una curva cerrada simple, por [2, Proposition 5.4, p. 11] $A$ es una curva cerrada libre. En el caso de que $A$ sea un arco libre con puntos finales $p$ y $q$, por el Teorema $4.5 p, q \notin \operatorname{int}(A)$.

Vol. 36, $\left.\mathrm{N}^{\circ} 2,2018\right]$ 
Para la suficiencia, supongamos que $A=X$ y $X$ es una curva cerrada. Es un resultado conocido que $C(X)$ es homeomorfo al disco unitario, y dado que $A$ está en el interior del disco unitario considerado como una variedad, tenemos que $A$ agujera a $C(X)$. Los dos casos restantes se siguen de los teoremas 4.1 y 4.6 .

En [4] los autores estudian los agujeros en dos tipos de continuos: estos son los abanicos suaves y los Elsa continuos.

Tenemos que un abanico $X$ con vértice $v$ se le llama suave si siempre que $\left\{x_{n}\right\} \rightarrow x$ en $X$, entonces $\left\{v x_{n}\right\} \rightarrow v x$ en $C(X)$. Además, decimos que un arco $A$ es un arco simple si $A \cap E(X)=\emptyset$, y si existe $\left\{A_{n}\right\}$ en $C(X)$ tal que $\left\{A_{n}\right\} \rightarrow A$ y para cada $n \in \mathbb{N}$ se cumple que $v \notin A_{n}, \operatorname{int}\left(A_{n}\right) \neq \emptyset$ y $A \cap A_{n} \neq \emptyset$.

El siguiente teorema da la clasificación de los elementos que agujeran a $C(X)$ cuando $X$ es un abanico suave.

Teorema 4.8 ([4, Theorem 3.8, p. 136]). Sea $X$ un abanico suave con vértice $v$ y $A \in$ $C(X)$. Entonces $A$ hace agujero en $C(X)$ si, y sólo si, $A$ es un arco simple.

Demostración. Para la necesidad. Sea $A$ un subcontinuo de $X$ y supongamos que $A$ agujera a $C(X)$. Por el Teorema 4.4 y por [4, Theorem 3.3, p. 134], tenemos que $A$ es un arco $p q$. Por $[4$, Theorem 3.6, p. 135] y [4, Theorem 3.7, p. 136], vemos que $p q \subset v e-\{v, e\}$, donde $e \in E(X)$. Además, qe es un arco libre en $X$. Con esto resulta fácil de ver que $A$ es un arco simple.

La suficiencia se sigue de [4, Theorem 3.5, p. 135].

El siguiente teorema da la clasificación de los elementos que agujeran a $C(X)$ cuando $X$ es un Elsa continuo.

Teorema 4.9 ([4, Theorem 4.4, p. 138]). Sea $X=J \cup R$ un Elsa continuo y sea $A \in C(X)$. Tenemos que $A$ agujera a $C(X)$ si, y sólo si, $A$ es un arco libre $p q$ tal que $p, q \notin \operatorname{int}(p q)$.

Demostración. Para la necesidad. Sea $A$ un subcontinuo de $X$ y supongamos que $A$ agujera a $C(X)$. Por el Teorema 4.4, por [4, Theorem 4.2, p. 137] y por [4, Theorem 4.3, p. 138], $A$ es un arco contenido en $R$. Así, $A$ es un arco libre en $X$. Por el Teorema 4.5 debemos tener que $p, q \notin \operatorname{int}(p q)$.

La suficiencia se sigue del Teorema 4.1.

\subsection{Para $F_{2}(X)$}

En [9] los autores estudian los agujeros en el segundo producto simétrico en dos tipos de continuos. El siguiente teorema da la clasificación de los elementos que agujeran a $F_{2}(X)$, cuando $X$ es una dendrita.

Teorema 4.10 ([9, Theorem 2.7, p. 86]). Sea $X$ una dendrita y sea $\{x, y\} \in F_{2}(X)$. Tenemos que $\{x, y\}$ hace agujero en $F_{2}(X)$ si, y sólo si, $x \neq y$ y ni $x$ ni $y$ son puntos finales de $X$, o si $x=y$ y $x$ es un punto de ramificación.

[Revista Integración, temas de matemáticas 
Demostración. Sean $x, y \in X$ tales que $\{x, y\}$ agujera a $F_{2}(X)$. Si $x=y$, por $[9$, Theorem 2.6, p. 86] y por $[9$, Corollary 2.5, p. 86] tenemos que $x$ es un punto de ramificación de $X$. Si $x \neq y$, entonces por [9, Corollary 2.5, p. 86] ninguno de los puntos pueden ser finales en $X$. El recíproco lo tenemos por $[9$, Theorem 2.1, p. 84] y $[9$, Theorem 2.2, p. 84]. $\square$

El siguiente teorema da la clasificación de los elementos que agujeran a $F_{2}(X)$ cuando $X$ es un abanico que es homeomorfo a un cono sobre un compacto.

Teorema 4.11 ([9, Theorem 3.10, p. 91]). Sea $X$ un abanico que es homeomorfo a un cono sobre un compacto, sea $\{x, y\} \in F_{2}(X)$ y sea $v$ el punto de ramificación. Tenemos que $\{x, y\}$ hace agujero en $F_{2}(X)$ si, y sólo si, $x=y=v$, o $y \neq x$ y $x, y \in C u t(X) \cup\{v\}$.

Demostración. Sean $x, y \in X$ tales que $\{x, y\}$ agujeran a $F_{2}(X)$. Si $x=y$, por $[9$, Theorem 3.4, p. 87] y [9, Theorem 3.8, p. 87] tenemos que $x=v$. Si $x \neq y$, entonces por $[9$, Theorem 3.6, p. 87] y $[9$, Theorem 3.9, p. 89] tenemos que $x, y \in C u t(X) \cup\{v\}$. El recíproco lo tenemos por [9, Theorem 3.2 , p. 87], [9, Theorem 3.3, p. 87] y [9, Theorem 3.5, p. 87].

En [10] los autores estudian los agujeros en el segundo producto cuando $X$ es un continuo unicoherente localmente conexo. Para la prueba de que este hiperespacio es unicoherente, ver [16, Theorem 1].

Teorema 4.12 ([10, Theorem 3.5, p. 258]). Sea $X$ un continuo unicoherente localmente conexo y sean $x, y \in X$. Tenemos que $\{x, y\}$ agujera a $F_{2}(X)$ si, y sólo si, ocurre una de las situaciones siguientes: $x=y$ y $X-\{x\}$ tiene al menos tres componentes; o $x \neq y$ y $x$ y y son puntos de corte de $X$.

Demostración. Sean $x, y \in X$ tales que $\{x, y\}$ agujeran a $F_{2}(X)$. Por [10, Theorem 3.3, p. 257] tenemos que $x, y \in C u t(X)$. Si $x=y$, por [10, Theorem 3.4, p. 257] vemos que $X-\{x\}$ tiene al menos tres componentes. El recíproco lo tenemos por [10, Theorem 3.1, p. 256] y [10, Theorem 3.2 , p. 256].

\subsection{Para $\operatorname{Cono}(X)$ y $\operatorname{Sus}(X)$}

En [5] los autores estudian los agujeros en el cono topológico y la suspensión topológica. Antes de enunciar los resultados demos la notación que se usa. Consideremos $Z$ como espacio métrico. El vértice del $\operatorname{Cono}(Z)$ se denota por $v$ y es el punto donde se identifica $Z \times\{1\}$. Un elemento de $\operatorname{Cono}(Z)-\{v\}$ será denotado por $[z, t]_{c}$, con $z \in Z$ y $t \in[0,1)$. La base del $C o n o(Z)$ es el conjunto $B(Z)=\left\{[z, 0]_{c}: z \in Z\right\}$. Para la $S u s(Z)$, el punto de identificación es denotado por $B_{Z}$. Para todo $[z, t]_{c} \in C o n e(Z)-\{B(Z), v\}$ su clase en $\operatorname{Sus}(Z)$ es denotada por $[z, t]_{s}$. Los resultados que los autores encuentran son los siguientes.

Proposición 4.13 ([5, Proposition 3.4]). Sea $Z$ un espacio métrico. Entonces ningún $z \in Z,[z, 0]_{c}$ agujera a $\operatorname{Cono}(Z)$.

Vol. 36, $\left.\mathrm{N}^{\circ} 2,2018\right]$ 
Demostración. Se puede probar con cierta facilidad que Cono $(Z)-\left\{[z, 0]_{c}\right\}$ es contráctil, y por la Proposición 3.4 tiene la propiedad b). Ahora, por [5, Proposition 3.2] este espacio es normal y por el Teorema 3.5 tenemos que es unicoherente.

Observación 4.14. Como una aplicación del resultado anterior consideremos a $I^{2}$. Es sabido que $I^{2} \approx \operatorname{Cono}\left(S^{1}\right)$, así que si $Z \approx I^{2}$ tenemos que para todo $z \in \partial(Z)$ se cumple que $Z-\{z\}$ es unicoherente y tiene la propiedad b), ya que es localmente conexo, donde $\partial(Z)$ denota la frontera de $Z$ vista como una variedad.

Proposición 4.15 ([5, Proposition 3.6]). Sea $Z$ un espacio métrico y supongamos que pq es un arco libre en $Z$ tal que $p, q \notin i n t(p q)$. Si $r \in p q$, entonces para todo $t_{0} \in(0,1)$ tenemos que $\left[r, t_{0}\right]_{c}$ agujera a Cono $(Z)$.

Demostración. Sea $r_{0} \in p q$ tal que $r_{0} \neq r$. Consideremos un arco $r r_{0}$ de $r$ a $r_{0}$ contenido en $p q$. Se consideran ahora los subconjuntos $\mathcal{C}_{1}=\left\{[s, t]_{c}: s \in r r_{0}, t \in\left[0, t_{0}\right]\right\}-\left\{\left[r, t_{0}\right]_{c}\right\}$ y $\mathcal{C}_{2}=\operatorname{cl}\left(\right.$ Cone $\left.(Z)-\mathcal{C}_{1}\right)-\left\{\left[r, t_{0}\right]_{c}\right\}$. Entonces $\mathcal{C}_{1} \cup \mathcal{C}_{2}=$ Cone $(Z)-\left\{\left[r, t_{0}\right]_{c}\right\}$ y $\mathcal{C}_{1} \cap \mathcal{C}_{2}=$ $\left\{[r, t]_{c}: t \in\left[0, t_{0}\right)\right\} \cup\left\{\left[r_{0}, t\right]_{c}: t \in\left[0, t_{0}\right]\right\} \cup\left\{\left[s, t_{0}\right]_{c}: s \in r r_{0}, s \neq r\right\}$, la cual no es conexa. Así, podemos concluir que $\left[r, t_{0}\right]_{c}$ agujera a $\operatorname{Cone}(Z)$.

Proposición 4.16 ([5, Proposition 3.7]). Sea $Z$ un espacio métrico y supongamos que pq es un arco libre en $Z$. Si $p \in$ int $(p q)$, entonces para todo $t_{0} \in(0,1)$ tenemos que $\left[p, t_{0}\right]_{c}$ no agujera a $\operatorname{Cono}(Z)$.

Demostración. Tenemos que Cone $(Z)$ tiene la propiedad b) por ser contráctil. Por otro lado, Cone $(p q)$ es una vecindad conexa de $\left[p, t_{0}\right]_{c}$ en $C o n e(Z)$ y bd $(C o n e(p q))$ es conexa. Por la Observación 4.14, Cone $(p q)-\left\{\left[p, t_{0}\right]_{c}\right\}$ tiene la propiedad b). Por lo tanto, por $\left[2\right.$, Theorem 2.4 , p. 3] tenemos que Cone $(Z)-\left\{\left[p, t_{0}\right]_{c}\right\}$ tiene la propiedad b), y por el Teorema 3.5 es unicoherente.

Proposición 4.17 ([5, Proposition 3.8]). Sean $Z$ un espacio métrico y $z_{0} \in Z$ tal que $Z-\left\{z_{0}\right\}$ es conexo. Si $Z$ tiene la propiedad b), entonces para todo $t_{0} \in(0,1)$ tenemos que $\left[p, t_{0}\right]_{c}$ no agujera a $\operatorname{Cono}(Z)$.

Demostración. Sea $z_{0} \in Z$ tal que $Z-\left\{z_{0}\right\}$ es conexo, y sea $t_{0} \in(0,1)$. La idea de la demostración es usar la Proposición 3.1. Los subconjuntos que se usan son $\mathcal{C}_{1}=$ $\left\{[z, t]_{c}: z \in Z, t \in\left[0, t_{0}\right]\right\}-\left\{\left[z_{0}, t_{0}\right]_{c}\right\}$ y $\mathcal{C}_{2}=\operatorname{cl}\left\{\right.$ Cone $\left.(Z)-\mathcal{C}_{1}\right\}-\left\{\left[z_{0}, t_{0}\right]_{c}\right\}$, los cuales son cerrados y conexos en $C o n e(Z)-\left\{\left[z_{0}, t_{0}\right]_{c}\right\}$. De estos podemos ver que $\mathcal{C}_{1} \cup \mathcal{C}_{2}=$ Cone $(Z)-\left\{\left[z_{0}, t_{0}\right]_{c}\right\}$ y $\mathcal{C}_{1} \cap \mathcal{C}_{2}=Z \times\left\{t_{0}\right\}-\left\{\left[z_{0}, t_{0}\right]_{c}\right\}$. Por hipótesis tenemos que $Z$ tiene la propiedad b), por la Proposición $3.3 \mathcal{C}_{1}$ tiene la propiedad b) y por la Proposición 3.4 $\mathcal{C}_{2}$ tiene la propiedad b). Como $\mathcal{C}_{1} \cap \mathcal{C}_{2}$ es conexo, podemos concluir la prueba.

Para la prueba de la siguiente proposición basta usar el Teorema 3.12.

Proposición 4.18 ([5, Proposition 3.9]). Sea $Z$ un espacio métrico. Si $Z$ no es unicoherente, entonces $v$ agujera a $C o n o(Z)$.

Los anteriores son los resultados que se presentan para el cono; para la suspensión son los siguientes.

[Revista Integración, temas de matemáticas 
Proposición 4.19 ([5, Proposition 3.10 and Proposition 3.11]). Para todo espacio métrico $Z$, ni $B_{Z}$ ni $v$ agujeran a $\operatorname{Sus}(Z)$.

Demostración. Haremos la prueba sólo para $B_{Z}$, ya que $S u s(Z)-\{v\} \approx S u s(Z)-B_{Z}$. Nótese que $\{v\}$ es un retracto por deformación de $C o n e(Z)-B(Z)$. Así, Cone $(Z)-$ $B(Z)$ es contráctil, por la Proposición 3.4 tiene la propiedad b) y por el Teorema 3.5 es unicoherente. El resultado se sigue del hecho de que Cone $(Z)-B(Z) \approx S u s(Z)-B_{Z}$. $\quad \nabla$

A diferencia del cono, si se cuenta con un teorema de clasificación para la suspensión, es el siguiente.

Teorema 4.20 ([5, Proposition 3.12]). Sean $Z$ un espacio métrico y $z \in Z$. Tenemos que $Z-\{z\}$ es conexo si, y sólo si, cualquiera que sea $t_{0} \in(0,1),\left[z, t_{0}\right]_{s}$ no agujera a $\operatorname{Cono}(Z)$.

Demostración. Supongamos que $Z-\{z\}$ es conexo. Para esta implicación se usa la Proposición 3.1 de la siguiente forma. Dado que $\{v\}$ es un retracto por deformación de $\mathcal{C}_{1}=$ $\left(Z \times\left[t_{0}, 1\right] / Z \times\{1\}\right)-\left\{\left[z, t_{0}\right]_{s}\right\}$ y $\left\{B_{Z}\right\}$, lo es para $\mathcal{C}_{2}=\left(Z \times\left[0, t_{0}\right] / B(Z)\right)-\left\{\left[z, t_{0}\right]_{s}\right\}$, y por la Proposición 3.4 ambos tienen la propiedad b). Como $\left(Z \times\left\{t_{0}\right\}\right)-\left\{\left[z, t_{0}\right]_{s}\right\} \approx Z-\{z\}$, entonces $\mathcal{C}_{1} \cap \mathcal{C}_{2}$ es conexo. Por lo tanto $\mathcal{C}_{1} \cup \mathcal{C}_{2}$ tiene la propiedad b).

Para la otra implicación, sean $z \in Z$ y $t_{0} \in(0,1)$ tales que $\left[z, t_{0}\right]_{s}$ no agujera a $\operatorname{Sus}(Z)$. Sean $\mathcal{C}_{1}=\left(Z \times\left[t_{0}, 1\right] / Z \times\{1\}\right)-\left\{\left[z, t_{0}\right]_{s}\right\}$ y $\mathcal{C}_{2}=\left(Z \times\left[0, t_{0}\right] / B(Z)\right)-\left\{\left[z, t_{0}\right]_{s}\right\}$. Ambos son subconjuntos cerrados y conexos de $\operatorname{Sus}(Z)-\left\{\left[z, t_{0}\right]_{s}\right\}$. Además, $\mathcal{C}_{1} \cup \mathcal{C}_{2}=\operatorname{Sus}(Z)-$ $\left\{\left[z, t_{0}\right]_{s}\right\}$. Así, por la unicoherencia de $\operatorname{Sus}(Z)-\left\{\left[z, t_{0}\right]_{s}\right\}$ tenemos que $\mathcal{C}_{1} \cap \mathcal{C}_{2} \approx Z-\{z\}$ es conexa.

Como una aplicación de los resultados obtenidos sobre el cono y la suspensión, en el mismo trabajo los autores dan teoremas de clasificación para el cono y la suspensión de las siguientes familias de continuos.

1. gráficas finitas (ver [5, Subsection 4.1]);

2. n-celdas y el cubo de Hilbert (ver [5, Subsection 4.2]);

3. variedades cerradas conexas y variedades cerradas conexas con frontera (ver $[5$, Subsection 4.3]);

4. Elsa continuos (ver [5, Subsection 4.4]), y

5. Elsa círculos y doble Elsa círculos (ver [5, Subsection 4.5]).

\subsection{Otros hiperespacios}

En esta sección presentamos los resultados que se conocen para otros hiperespacios diferentes a los que ya se mencionaron.

Sabemos que si $X$ es localmente conexo, entonces $2^{X} \approx I^{\infty}$ (ver [20, Theorem 11.3, p. 89]). También sabemos que si $X$ es localmente conexo sin $\operatorname{arcos}$ libres, entonces $C_{n}(X) \approx$

Vol. 36, $\mathrm{N}^{\circ}$ 2, 2018] 
$I^{\infty}$ (ver [24, Theorem 7.1, p. 250]). En [5, Lemma 4.9] los autores prueban que en el cubo de Hilbert ningún elemento lo agujera, así que tenemos los siguientes dos teoremas.

Teorema 4.21 ([5, Theorem 5.1]). Si $X$ es un continuo localmente conexo, entonces ningún $A \in 2^{X}$ agujera a $2^{X}$.

Teorema 4.22 ([5, Theorem 5.2]). Si $X$ es un continuo localmente conexo sin arcos libres, entonces ningún $A \in C_{n}(X)$, cualquiera que sea $n \in \mathbb{N}$, puede agujerar a $C_{n}(X)$.

Un resultado que presentan los autores en este trabajo es el siguiente.

Teorema 4.23 ([5, Theorem 5.5]). Sea $Y$ un espacio métrico compacto. Consideremos $X=\operatorname{Cono}(Y)$ y a $\mathcal{H}(X) \in\left\{2^{X}, C_{n}(X), F_{n}(X)\right\}$, para $n \in \mathbb{N}$. Entonces $\mathcal{H}(X) \approx$ $C o n o(Z)$, donde $Z$ es un continuo.

Para finalizar damos un corolario, consecuencia de este interesante resultado. Nótese que la prueba es una consecuencia directa de la Proposición 4.13.

Corolario 4.24 ([5, Corollary 5.6]). Sea $Y$ un espacio métrico compacto. Consideremos $X=\operatorname{Cono}(Y)$ y a $\mathcal{H}(X) \in\left\{2^{X}, C_{n}(X), F_{n}(X)\right\}$, para $n \in \mathbb{N}$. Si $A \in Z=\{A \in \mathcal{H}(X)$ : $A \cap B(Y) \neq \emptyset\}$, entonces $A$ no agujera a $\mathcal{H}(X)$. En particular, $X$ no agujera a $\mathcal{H}(X)$.

\subsection{Conclusiones}

Hemos presentado los resultados que son el inicio de esta área de estudio, la cual continúa activamente. Por ejemplo, podemos mencionar dos trabajos más que están por aparecer. El primero es el estudio de los agujeros en el hiperespacio suspensión $H(X)=C(X) / F_{1}(X)$, ver [6]. El segundo aborda este problema para $C(X)$ de dendroides suaves, ver [3]. La existencia de otras clases de continuos y de otros tipos de hiperespacios, abre una gran gama de posibles objetos de estudio. Aunada a todo esto, tenemos la posibilidad de estudiar los agujeros respecto al grado de multicoherencia (ver [27, Section 13.32, p. 292]) de un espacio topológico dado. Ejemplos de esto son los trabajos [7] y [8]. En [7] los autores estudian los agujeros respecto al grado de multicoherencia en el segundo producto simétrico de los subcontinuos del continuo conocido como Figura 8. En [8] los autores dan un teorema de clasificación de los agujeros respecto al grado de multicoherencia del segundo producto simétrico de gráficas conexas cíclicas.

Agradecimientos. Los autores agradecemos el tiempo dedicado y las atinadas observaciones hechas por el (la) árbitro, quien indicando errores e imprecisiones ayudó con la versión definitiva de este artículo.

\section{Referencias}

[1] Anaya J.G., "Making holes in hyperspaces", Topology Appl. 154 (2007), 2000-2008.

[2] Anaya J.G., "Making holes in the hyperspaces of a Peano continuum", Topology Proc. 37 (2011), 1-14.

[Revista Integración, temas de matemáticas 
[3] Anaya J.G., Carranza R.I., Maya D. and Orozco-Zitli F., "Making holes in the hyperspace of subcontinua of smooth dendroids", Preprint.

[4] Anaya J.G., Castañeda-Alvarado E. and Orozco-Zitli F., "Making holes in the hyperspace of subcontinua of some continua", Adv. in Pure Math. 2 (2012), 133-138.

[5] Anaya J.G., Castañeda-Alvarado E., Fuentes-Montes de Oca A. and Orozco-Zitli F., "Making holes in the cone, suspension and hyperspaces of some continua", Comment. Math. Univ. Carolin. 59 (2018), No. 3, 343-364.

[6] Anaya J.G., Castañeda-Alvarado E., Fuentes-Montes de Oca A. and Orozco-Zitli F., "Making holes in the hyperspaces suspension of some continua", Preprint.

[7] Anaya J.G., Maya D. y Orozco-Zitli F., "Agujeros en el segundo producto simétrico de subcontinuos del continuo Figura 8", CIENCIA ergo-sum 17 (2010), No. 3, 307-312.

[8] Anaya J.G., Maya D. and Orozco-Zitli F., "Making holes in the second symmetric products of a cyclicly connected graph", Journal of Mathematics Research 6 (2014), No. 3, 105-113.

[9] Anaya J.G., Maya D. and Orozco-Zitli F., "Making holes in the second symmetric products of dendrites and some fan", CIENCIA ergo-sum 19 (2012), No.1, 83-92.

[10] Anaya J.G., Maya D. and Orozco-Zitli F., "Making holes in the second symmetric product of unicoherent locally connected continua", Topology Proc. 48 (2016), 251-259.

[11] Borsuk K., "Quelques théorémes sur les ensembles unicoherents", Fund. Math. 17 (1931), No.1, 171-209.

[12] Castañeda E., "A Unicoherent Continuum Whose Second Symmetric Product is not Unicoherent", Topology Proc. 23 (1998), 61-67.

[13] Eilenberg S., "Sur les transformations d'espaces métriques en circonférence", Fund. Math. 24 (1935), No.1, 160-176.

[14] Eilenberg S., "Transformations continues en circonférence et la topologie du plan", Fund. Math. 26 (1936), No. 1, 61-112.

[15] Ganea T., "Covering spaces and cartesian products", Ann. Soc. Polon. Math. 25 (1952), $30-42$.

[16] Ganea T., "Symmetrische Potenzen topologischer Raume", Math. Nachr. 11 (1954), No. 4-5, 305-316.

[17] García-Máynez A. and Illanes A., "A survey on unicoherence and related properties", An. Inst. Mat., Univ. Nac. Autón. Méx. 29 (1989), 17-67.

[18] Illanes A., "Multicoherence of Whitney levels", Topology Appl. 68 (1996), No.3, 251-265.

[19] Illanes A., "The hyperspace $C_{2}(X)$ for a finite graph $X$ is unique", Glas. Mat. Ser. III 37 (2002), 347-363.

[20] Illanes A. and Nadler S. B., Jr., Hyperspaces, Fundamentals and Recent Advances, Monographs and Textbooks in Pure and Applied Mathematics, 216, Marcel Dekker, New York, 1999.

[21] Kuratowski K., "Sur les continus de Jordan et le théoréme de M. Brouwer", Fund. Math. 8 (1926), No. 1, 137-150.

Vol. 36, $\left.\mathrm{N}^{\circ} 2,2018\right]$ 
[22] Kuratowski K., "Une carecterisation topologique de la surface de la sphére", Fund. Math. 13 (1929), No. 1, 307-318.

[23] Macías S., "On symmetric products of continua", Topology Appl. 92 (1999), No. 2, 173-182.

[24] Macías S., "On the hyperspaces $C_{n}(X)$ of a continuum X", Topology Appl. 109 (2001), $237-256$.

[25] Mardešić S., "Equivalence of singular and Čech homology for ANR-s. Application to unicoherence", Fund. Math. 46 (1958), No. 1, 29-45.

[26] Nadler Jr. S.B., "Arc components of certain chainable continua", Canad. Math. Bull. 14 (1971), 183-189.

[27] Nadler Jr. S.B., Continuum Theory, An Introduction, Monographs and Textbooks in Pure and Applied Mathematics, 158, Marcel Dekker, Inc., New York, 1992.

[28] Nadler Jr. S.B., "Continua whose cone and hyperspace are homeomorphic", Trans. Amer. Math. Soc. 230 (1977), 321-345.

[29] Nadler Jr. S.B., Hyperspace of sets, Monographs and Textbooks in Pure and Applied Mathematics, 49, Marcel Dekker, New York, 1978.

[30] Whyburn G.T., Analytic Topology, AMS Colloquium Publications, 28, Providence, R.I., 1942. 